Atkins, L; Michie, S; (2013) Changing eating behaviour: What can we learn from behavioural science? Nutrition Bulletin, 38 (1) pp. 30-35. 10.1111/nbu.12004. Downloaded from UCL Discovery: http://discovery.ucl.ac.uk/1400697/.

\title{
ARTICLE
}

\section{Changing eating behaviour: what can we learn from behavioural science?}

Dr Lou Atkins ${ }^{1, *}$

Professor Susan Michie ${ }^{1}$

${ }^{1}$ Centre for Research Outcomes and Effectiveness, Research Department of Clinical,

Education and Health Psychology, University College London, 1-19 Torrington Place,

London WC1E 7 HB

* Corresponding Author: Dr Lou Atkins; Telephone: 0207679 1977; E-mail:

l.atkins@ucl.ac.uk.

\section{Summary}

Designing effective behaviour change interventions is key to promoting healthy eating. This paper takes a theoretical perspective to consider why people don't behave in ways that promote health, and summarises evidence-based principles and strategies for behaviour change. We present a theoretically-driven, comprehensive method for intervention design using several tools recently developed in behavioural science.

Keywords: Eating, behaviour change, behavioural science, behaviour change interventions, behaviour change techniques, Behaviour Change Wheel.

\section{Introduction}

Obesity is one of the main health risk factors in the UK today. Prevalence has doubled in the last 25 years (Lobstein and Leach 2007) with $26 \%$ of adults and $16 \%$ children in the UK classed as obese (The NHS Information Centre 2012). In addition to the well-documented health costs associated with obesity, such as cardiovascular disease, stroke and diabetes, it has major financial implications. In the UK alone, costs attributable to obesity are estimated to be around $£ 5$ billion per year ( $\mathrm{DH}$ 2011). With purchases of fruit and vegetables falling by $12 \%$ and $3 \%$ respectively in the last five years (The NHS Information Centre 2012) promoting healthy eating is key to addressing the obesity epidemic. But in an 'obesogenic environment' how can this be done?

In this article we discuss the contribution of behavioural science to our understanding of why people don't behave in ways that promote health, in particular eating healthily; and how behavioural science can help us to design more effective interventions to encourage dietary behaviour change.

\section{Why don't we behave in ways that promote health?}

Theories in behavioural science have contributed substantially to our understanding of behaviour. For example, the 'COM-B' model of behaviour (Michie et al. 2011c) recognises that for any behaviour to occur, the individuals concerned must have the physical and psychological capability to enact it, the physical and social opportunity, and be motivated to do it more than any potentially competing behaviour on relevant occasions. Thus, they must be clear about how to do it, understand why it is important and have the necessary skills to facilitate change. They must also have their social and physical environment set up to ensure that they have everything to hand when needed. They must have the time available and there may be a need for cues and prompts to remind them. They must be convinced 
that it is worthwhile and prioritise it when required. Viewing it as a norm among their peer group is also often very important.

Considering motivation to change in more detail, it is clear that it involves both reflective processes (i.e. the self-conscious intentions or plans we make and the beliefs we hold) and automatic processes (i.e. our wants, needs and impulses). Our intentions and plans can only influence behaviour if they generate sufficiently strong wants or needs at the relevant moment to overcome competing wants or needs (West 2006). Looking at motivation and behaviour in this way allows us to understand why intentions often don't translate into behaviour. We might plan to eat a healthy meal and fully intend to choose the salad rather than the burger in the canteen at lunch but if there is a stronger competing desire (e.g. triggered by the smell of the burger on the grill etc.) our intentions will lose out to these desires.

\section{Principles guiding intervention design}

Health promotion approaches to changing behaviour have focussed on giving information and largely ignored the role of motivational, social and environmental factors. Intervention designers have made use of 'fear appeals' (i.e. provoking fear to encourage behaviour change such as showing graphic images of clogged arteries in stop smoking campaigns). There has been limited evidence for the effectiveness of these approaches (Milne et al. 2000;Witte and Allen 2000; Brewer et al. 2007) and more comprehensive interventions that take account of both intra-psychic and external factors have been shown to be more effective (NICE 2007).

\section{NICE guidelines for intervention design}

Evidence-based guidance for policy makers and practitioners in changing health behaviours is provided in the National Institute for Health and Clinical Excellence (NICE) 'Guidelines for Behaviour change at population, community and individual levels' (NICE 2007). These guidelines describe several theory and evidence-based principles of changing behaviour at the individual level, relevant to regulating eating behaviour. These are summarised below (box 1) and have been grouped according to a theoretically-based model comprising three essential conditions for behaviour to occur: Capability, Opportunity and Motivation (COM-B model) (Michie et al. 2011c). We will return to discuss this model later.

\section{Box 1. Summary of principles of individual behaviour change (NICE, 2007)}

Capability

- Help individuals to understand the health consequences of their behaviours, e.g. effect of a high saturated fat diet on cardiovascular functioning

- Encourage forming goals and specific plans to initiate change, e.g. planning to eat a salad for lunch at work every day next week

- Help people develop strategies to cope with difficult situations where risk of relapse is elevated, e.g. dealing with the situation of being offered cake at someone's house

\section{Opportunity}

- Encourage entering in to 'behavioural contracts' with others, e.g. agreeing with a friend not to eat chocolate for the next three months

- Develop understanding of how the social environment might affect behaviour, e.g. exploring how going out for lunch with friends might affect plans to eat healthily

\section{Motivation}

- Emphasise the personal relevance of health behaviours, e.g. what eating healthily would mean to the individual

- Promote positive attitudes towards the consequences of changing behaviour, e.g. 


\section{emphasising how good the person will feel if they adopt a low fat diet}

- Promote visibility of positive examples of others behaviour and approval of others for these behaviours, e.g. conveying that others eat healthily and would be happy to see them eating healthily

- Enhancing individuals beliefs about their ability to change, e.g. telling someone they have the ability to change how they eat

One of the key recommendations of the House of Lords Science and Technology Select Committee's (HLSTSC) report on behaviour change (HLSTSC 2011) was for NICE to update these guidelines to reflect the evidence amassed since they were published half a decade ago. This is currently in progress and new guidance is due in December 2013.

\section{Recent evidence}

Since the publication of NICE public health guidance, work has been done to synthesise existing evidence on the effectiveness of healthy eating interventions within theoretical frameworks to try and identify effective individual, and clusters of, behaviour change techniques (BCTs). This work has been made possible by the development of taxonomies of BCTs that provide consensus-based, standard terminology to reliably identify and describe intervention content.

Michie and colleagues conducted a meta-regression of 122 evaluations of interventions to promote physical activity and healthy eating in the general population. Using a taxonomy of 26 BCTs (Abraham and Michie 2008) developed for this purpose, they grouped BCTs according to the constructs comprising Control Theory (Carver and Scheier 1982). The central tenet of Control Theory is that setting goals, monitoring behaviour, receiving feedback, and reviewing relevant goals in the light of feedback are key to self-management and behavioural control. They found that interventions including the BCT 'self-monitoring' (defined as establishing a method to record a behaviour or outcome of a behaviour, e.g. keeping a food diary or recording weight) and a least one other BCT predicted by Control Theory to operate synergistically were twice as effective as other interventions (Michie et al. 2009).

Using the same taxonomy, Dombrowski and colleagues reviewed 44 evaluations of interventions aimed at promoting healthy eating and/or physical activity in obese adults with obesity-related comorbidities or additional risk factors for co-morbidities to identify effective BCTs. They also found that interventions that were theoretically coherent in terms of Control Theory were more effective than those that were not. Like Michie and colleagues, they found that interventions with more BCTs congruent with Control Theory, in particular selfmonitoring of dietary behaviour, resulted in a significant increase in weight loss. They also reported that interventions including the BCTs 'provision of dietary instructions' and 'relapse prevention' were effective in promoting weight loss (Dombrowski et al. 2012).

These reviews not only identify effective BCTs and theoretical frameworks to guide the design of interventions to promote healthy eating; they also demonstrate the value of theoretically-driven syntheses of evidence to identify active ingredients that can be used in intervention design.

\section{A theoretically-driven approach to intervention design}

MRC's guidance for designing and evaluating complex interventions (Craig et al. 2008) recommend taking a theoretically-based approach to intervention design. However, there is no detail as to how to do this. We present here four recently developed behavioural science technologies and show how they interlink and can be applied to understanding behaviour and to designing and evaluating behaviour change interventions.

These technologies are the COM-B model (Michie et al. 2011c) and Theoretical Domains Framework (Michie et al. 2005; Cane et al. 2012) to guide understanding of behaviour and; 
the Behaviour Change Wheel (Michie et al. 2011c) and Behaviour Change Techniques Taxonomy (v1) (Michie et al. 2011a) to guide the development and specify content of behaviour change interventions. These tools interlink to form six steps to intervention design:

\section{Step 1: Selecting the target behaviour}

Behaviour does not exist in a vacuum but occurs within a context and within a system of behaviours. This system of behaviours might occur at different levels. For example, in promoting healthy eating, interventions could target one or more of the following levels: Government policy makers (e.g. decisions about pricing, food labelling, advertising); Supermarket managers (e.g. product placement, promotions); Shoppers (e.g. menu planning, list making, food selection); those involved in food preparation (e.g. menu planning, food selection, portions); and those consuming the food (e.g. rule-making, food selection, portions). In selecting which behaviour to target, designers should list all the behaviours that can potentially play a role in bringing about the desired change then select one to target in the first instance, guided by how central it is to bringing about change and how easy it is to change.

Step 2: Specifying the target behaviour

Once the target behaviour has been selected, it then needs to be described in as much detail as possible, i.e. describing who needs to do what, when, where, how often, with whom and in what context. The reason for this is that vaguely specified target behaviours, for example, 'eating less' will not provide the behavioural specificity required for an informative behavioural analysis; preferable would be a target of 'consuming xx fewer calories per day'. More precisely specified behaviours are also easier to measure and therefore provide a baseline and metric for evaluating the success of an intervention.

Step 3: Understanding the target behaviour

When the target behaviour has been specified as precisely as possible designers need to undertake a behavioural analysis to understand why behaviours are as they are and what needs to change in order to bring about the desired behaviour.

The COM-B model and TDF provide a method for understanding the target behaviour theoretically and are the starting point for intervention design. The COM-B model allows a simple approach to understanding behaviour in context in terms of the Capability, Opportunity and Motivation needed for a behaviour to occur. It may be that an individual is highly motivated to engage in the desired behaviour (e.g. eating healthily) but does not have the capability (e.g. ability to cook healthy food) or the opportunity (e.g. is not responsible for the shopping). The TDF is an elaboration of the COM-B model, with 14 domains of theoretical constructs that map on to the COM-B components and allow for a more detailed understanding of behaviour (box 2). Questions for use in interviews or focus groups have been developed to gather data about behaviour. For example, to explore the domain 'behavioural regulation' (i.e. planning and monitoring behaviour), respondents could be asked "What preparatory steps are needed to do x?"(Michie et al. 2005). In addition to covering internal factors, the domains cover contextual and organisational aspects, such as the role of social influences (i.e. interpersonal processes that can cause individuals to change their thoughts, feelings, or behaviours) and the environmental context (i.e. circumstances in individuals' situation or environment that discourage or encourage the development of skills and abilities, independence, social competence, adaptive behaviour and physical environment). 
Box 2. Mapping COM-B components to TDF domains

\begin{tabular}{|c|c|c|}
\hline COM-B com & ponent & TDF Domain \\
\hline Capability & Psychological & Knowledge \\
\hline & & Skills \\
\hline & & $\begin{array}{l}\text { Memory, attention and decision } \\
\text { processes }\end{array}$ \\
\hline & & Behavioural regulation \\
\hline & Physical & Skills \\
\hline Opportunity & Social & Social influences \\
\hline & Physical & $\begin{array}{l}\text { Environmental context and } \\
\text { resources }\end{array}$ \\
\hline Motivation & Reflective & Social/ professional role and identity \\
\hline & & Beliefs about capabilities \\
\hline & & Optimism \\
\hline & & Beliefs about consequences \\
\hline & & Intentions \\
\hline & & Goals \\
\hline & Automatic & Social/ professional role and identity \\
\hline & & Optimism \\
\hline & & Reinforcement \\
\hline & & Emotion \\
\hline
\end{tabular}

Step 4: Building the intervention

Having identified which domains within COM-B or TDF need to change to bring about the desired behaviour, designers have a basis for considering which intervention functions are most likely to be effective in changing the target behaviour in context. A comprehensive range of possible intervention functions is incorporated into the Behaviour Change Wheel (BCW), which is a synthesis of 19 behaviour change frameworks identified in a systematic review. It includes a range of frameworks from public health, culture change and environmental policy such as Intervention Mapping (Bartholomew et al. 2011) and MINDSPACE (Institute for Government 2010). The BCW provides a systematic approach to selecting from a comprehensive range of intervention functions: education, persuasion, incentivisation, coercion, training, restriction, environmental restructuring, modelling, and enablement. Through mapping work between the COM-B, TDF and BCW, designers can select an appropriate mix from the nine intervention functions based on the behavioural analysis (undertaken in step 3).

Step 5: Specifying intervention content

After identifying candidate intervention functions, the next step is to consider which BCTs to use to deliver each intervention function. Expert consensus work has built on previous taxonomies developed in specific behavioural domains (i.e. healthy eating, physical activity and smoking) to produce an extensive, reliable and generalisable set of BCTs for use in specifying, evaluating and implementing behaviour change interventions (Michie et al. 2011a).

Step 6: Delivering the intervention

Just as for intervention content, it is important to consider the full panoply of modes available for delivering interventions. Interventions can be delivered face-to-face to individuals or groups or remotely either through mass media (i.e. the internet, TV, radio, print media) or at the individual level through telephone support lines, text or smartphone apps.

In selecting the preferred mode or modes, designers should consider the evidence of effectiveness of modes of delivery, their local relevance, the practicability, affordability and public, professional and political acceptability of the mode of delivery. 
Finally, designers need to consider whether there are policies that would support the delivery of the intervention. The BCW suggests which policy categories are potentially appropriate to select according to the intervention functions comprising the intervention (i.e. communication/marketing, guidelines, fiscal, regulation, legislation, environmental/social planning, and service provision). The factors considered in relation to mode of delivery should also be considered in relation to policy selection and development, where appropriate.

This method of intervention design is being developed into a practical step-by-step guide for a wide range of audiences, including policy makers, intervention designers, researchers and clinical and public health practitioners (Michie et al., in preparation). A workshop for intervention designers using this approach is being developed in parallel and will accompany the step-by-step guide.

Where is this approach being applied?

This approach to intervention design is currently being applied in different areas of nutrition research, for example, a primary care intervention to reduce cardiovascular disease in people with severe mental illness ${ }^{1}$ and a smartphone app for parents to manage their children's weight ${ }^{2}$.

\section{How can we optimise learning from interventions to promote healthy eating?}

Better evaluations

Evaluation plans for interventions should ideally be developed as the intervention is designed. This is so that they include a study design that allows causal inference and that all components of the intervention and hypothesised causal mechanisms are measured. For optimisation of interventions, we need both evidence of effectiveness and evidence as to which components are effective and how they interact with other components and contribute to the outcome.

Interventions to promote healthy eating are complex with many interacting components and their effects are variable (NICE 2007). To understand this variation we need to conduct detailed analyses of mechanisms of action (examination of the moderating and mediating role of a range of variables) and interpret these within theoretical frameworks. Use of theoretical frameworks allows accumulation of evidence across individual studies.

\section{Better reporting}

What we can learn from published literature about behaviour change is limited by suboptimal reporting of the content of interventions. In comparison with the literature on pharmacological interventions where the formula, dosage and mechanism of action are usually precisely reported, the content of behaviour change interventions is rarely fully or consistently reported (Glasziou et al. 2008). Guidance such as the CONSORT (Consolidated Standards of Reporting Trials) (Boutron et al. 2008) and TREND (Transparent reporting of evaluations of nonrandomised designs) (Des Jarlais et al. 2004) statements have been developed to promote more complete reporting. More recently journals such as Implementation Science and Addiction have signed up to reporting convention agreements such as the WIDER (Workgroup for Intervention Development and Evaluation Research) recommendations to improve reporting not only of content but also the context and mode of delivery of interventions (WIDER 2009).

The development of taxonomies, specifically for interventions to promote physical activity and healthy eating like the CALO-RE taxonomy (Abraham \& Michie 2008; Michie et al. 2011b) and the comprehensive, generic taxonomy described earlier (Michie et al. 2011a)

\footnotetext{
${ }^{1}$ For more information contact Alex Burton, UCL (a.burton@ucl.ac.uk)

2 For more information contact Kristina Curtis, University of Warwick (k.e.curtis@warwick.ac.uk)
} 
give a common language for reporting intervention content to facilitate replication and implementation.

\section{Conclusion}

Theory and evidence-based tools developed in behavioural science play an important role in informing the design and evaluation of effective interventions to promote healthy eating behaviour. In order to build the evidence base of what works, when, in which context and for whom; interventions to promote healthy eating should be theoretically based, systematically designed, robustly evaluated and precisely reported.

\section{References}

Abraham C \& Michie S (2008) A taxonomy of behavior change techniques used in interventions. Health Psychology 27: 379-387.

Bartholomew L, Parcel G, Kok G et al. (2011) Planning Health Promotion Programmes: An Intervention Mapping Approach, 3rd ed. Jossey Bass.

Boutron I, Moher D, Altman DG et al. (2008) Extending the CONSORT statement to randomized trials of nonpharmacologic treatment: explanation and elaboration. Annals of Internal Medicine 148: 295-309.

Brewer NT, Chapman GB, Gibbons FX et al. (2007) Meta-analysis of the relationship between risk perception and health behavior: the example of vaccination. Health Psychology 26: 136-145.

Cane J, O'Connor D \& Michie S (2012) Validation of the theoretical domains framework for use in behaviour change and implementation research. Implementation Science, 7: 37 doi:10.1186/1748-5908-7-37.

Carver CS \& Scheier MF (1982) Control theory: A useful conceptual framework for personality-social, clinical, and health psychology. Psychological Bulletin 95: 111-135.

Craig P, Dieppe P, Macintyre $S$ et al. (2008) Developing and evaluating complex interventions:

the new Medical Research Council guidance. BMJ 337: 979-983.

DH (Department of Health) (2011) Healthy Lives, Healthy People: A call to action on obesity in England. Department of Health, London.

Des Jarlais DC, Lyles C, Crepaz N et al. (2004) Improving the reporting quality of nonrandomized evaluations of behavioral and public health interventions: the TREND statement. American Journal of Public Health 94: 361-366.

Dombrowski S, Sniehotta F, Avenell A et al. (2012) Identifying active ingredients in complex behavioural interventions for obese adults with obesity-related comorbidities or additional risk factors for co-morbidities: a systematic review. Health Psychology Review 6: 7-32.

Glasziou P, Meats E, Heneghan C et al. (2008) What is missing from descriptions of treatment in trials and reviews? BMJ 336: 1472-1474.

HLSTSC (House of Lords Science and Technology Select Committeee) (2011) Behaviour

Change, The Stationery Office Limited, London. Available at: http://www.publications.parliament.uk/pa/ld201012/ldselect/ldsctech/179/179.pdf (accessed 30 August 2011)

Institute for Government (2010) MINDSPACE: Influencing Behaviour Through Public Policy. Available http://www.instituteforgovernment.org.uk/sites/default/files/publications/MINDSPACE.pdf (accessed 15 November 2011).

Lobstein T, \& Leach T (2007) Tackling Obesities: Future Choices - International Comparisons of Obesity Trends, Determinants and Responses -Evidence Review. Foresight, Government Office of the Chief Scientist. Available at: http://www.dti.gov.uk/assets/foresight/docs/obesity/06\%20part\%2002.pdf (accesed 15 November 2012).

Michie S Abraham C, Whittington C et al. (2009) Effective techniques in healthy eating and physical activity interventions: a meta-regression. Health Psychology 28: 690-701 
Michie S, Johnston M Abraham C et al. (2005) Making psychological theory useful for implementing evidence based practice: a consensus approach. BMJ Quality \& Safety 14: 2633.

Michie S, Abraham C, Eccles M et al. (2011a) Strengthening evaluation and implementation by specifying components of behaviour change interventions: a study protocol. Implementation Science 6: 10 doi:10.1186/1748-5908-6-10.

Michie S, Ashford S, Sniehotta F et al. (2011b) A refined taxonomy of behaviour change techniques to help people change their physical activity and healthy eating behaviours: The CALO-RE taxonomy. Psychology \& Health 26: 1479-1498.

Michie S, van Stralen M \& West R (2011c) The behaviour change wheel: A new method for characterising and designing behaviour change interventions. Implementation Science 6: 42 doi:10.1186/1748-5908-6-42

Michie S, Atkins L \& West R (In preparation) The Behaviour Change Wheel (BCW) Guide to Designing Theory-based Interventions (working title).

Milne S, Sheeran P \& Orbell S (2000) Prediction and Intervention in Health-Related Behavior: A Meta-Analytic Review of Protection Motivation Theory. Journal of Applied Social Psychology 30: 106-143.

NICE (National Institute for Health and Clinical Excellence) (2007) Behaviour change at population, community and individual levels. NICE Public Health Guidance 6. National Institute for Health and Clinical Excellence, London.

NHS (National Health Service) Information Centre (2012) Statistics on obesity, physical activity and diet: England, 2012. Available at: http://www.ic.nhs.uk/webfiles/publications/003_Health_Lifestyles/OPAD12/Statistics_on_Ob esity_Physical_Activity_and_Diet_England_2012.pdf (accessed 16 November 2012). West R (2006) Theory of Addiction. Oxford, Wiley-Blackwell.

Witte K \& Allen M (2000) A Meta-Analysis of Fear Appeals: Implications for Effective Public Health Campaigns. Health Education \& Behavior 27: 591-615.

WIDER (Workgroup for Intervention Development and Evaluation Research) (2009) WIDER recommendations. Available at:

http://interventiondesign.co.uk/wp-content/uploads/2009/02/widerrecommendations.pdf (accessed 26 May 2011). 